\title{
Dynamic Data Modeling of SCR Denitration System Based on Mutual Information
}

\author{
Wenjie Zhao* and Luyao Zhang \\ Department of Automation, North China Electric Power University, Baoding, Hebei Province, China \\ ${ }^{*}$ Corresponding author
}

\begin{abstract}
The establishment of accurate models is vital in parametric optimization of control systems, and the choice of input variables can directly affect the accuracy and complexity of the model. Therefore, this paper proposed a modeling method based on mutual information (MI) and least squares support vector machine (LSSVM). On the basis of MI, the problem of delay, the correlation and redundancy among variables were considered synthetically. The optimal ones were screened through field measured variables by MI, and chosen as the input of LSSVM for predictions of output NOx concentration. The results proved the method can decrease complexity, improve approximation and generalization capability.
\end{abstract}

Keywords-coal-fired unit; SCR de-NOx system; mutual information; least squares support vector machine

\section{INTRODUCTION}

SCR denitrification (de-NOx) technology has been essential due to growth of demand for environmental protection. A model of SCR de-NOx system with good precision can be very important to optimize the control strategy of de-NOx system and improve the field control effect.

Numerous studies have developed models to predict the output NOx concentration. They are divided into two main groups: mechanism modeling and experimental modeling. Mechanism modeling methods expounded well, since they adoptd partial differential equations to describe the SCR denitrification process of mass and energy, and the mechanism of Eley-Rideal was generally considered suitable for the process of SCR de-NOx [1]. However, these methods were accused to be not suitable for every model, furthermore, analysis and formula deductions were considered to be quite intricate. Among experimental modeling methods, Artificial Neural Networks (ANN) and Support Vector Machine ( SVM ) have come into wide use in this area. They had strong nonlinear modeling capabilities, and were applicable to the case of complex systems and processes. Reference [2] combined ANN and genetic algorithm (GA) to model the characteristics of NOx emission and carbon combustion. References [3,4] used support vector machine (SVM) and least square support vector machine (LSSVM) respectively to establish the boiler combustion models and predict emissions of NOx. The major problems encountered above were adjusting parameters and selecting comprehensive data, since these parameters can affect modeling accuracy, and if input variables were unable to excite the entire internal state of the system, a high-precision model can not be obtained.
In addition, these models ignored time delay and the importance of interdependency between variables, and were only applied to steady-state models, which had some limitations and were difficult to use in the design of control strategies.

In view of the shortcomings above, a dynamic modeling method of LSSVM based on MI was proposed. MI had good performs in correlation analysis and feature selection $[5,6]$. LSSVM had strong generalization and approximation abilities, including reducing the risk of overfitting in modeling and generating models for the small number of records as well $[4,7]$.

In the model, MI was used through field measured variables to detect interactions, select appropriate delay and eliminate redundancy. The optimal ones were chosen as the input of LSSVM for predictions of output NOx concentration. The results showed that the method proposed in this paper was of high prediction accuracy.

\section{SCR DENITRIFICATION SYSTEM}

The system adoptd the fly-ash-rich arrangement (the SCR de-NOx reactor in the middle of economizer and air preheater). The flue gas from the economizer was reacted with diluted ammonia in the SCR denitrification reactor, and redoxed to $\mathrm{H}_{2} \mathrm{O}$ and $\mathrm{N}_{2}$ in the presence of catalysts. And the schematic illustration is shown in Figure 1. As most of the NOx in the boiler flue gas exists in the form of $\mathrm{NO}$, the main reactions are as follows:

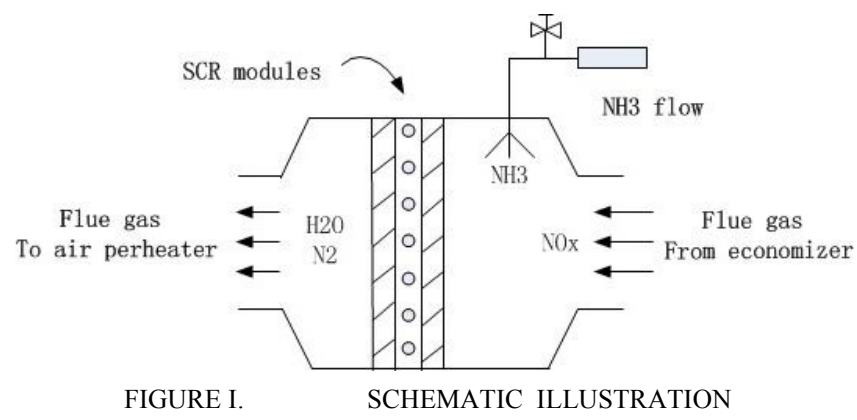

$$
4 \mathrm{NH}_{3}+4 \mathrm{NO}+\mathrm{O}_{2} \rightarrow 4 \mathrm{~N}_{2}+6 \mathrm{H}_{2} \mathrm{O}
$$

$$
4 \mathrm{NH}_{3}+6 \mathrm{NO} \rightarrow 5 \mathrm{~N}_{2}+6 \mathrm{H}_{2} \mathrm{O}
$$




\section{VARIABLE SELECTION}

\section{A. Mutual Information}

Mutual information is used to evaluate the amount of information that a event contributes to another, and is widely used in feature selection. MI is a nonnegative number $(I(x, y)$ $\geq 0$ ), and a value of zero denotes irrelevance.

The MI between $\mathrm{x}$ and $\mathrm{y}$ is defined by the following formula:

$$
I(x, y)=\int_{y} \int_{x} p(x, y) \log \frac{p(x, y)}{p(x) p(y)} .
$$

If $x$ and $y$ are random variables, $p(x)$ and $p(y)$ are probability density functions, and $p(x, y)$ represents the joint probability of a given stimulus.

Conditional mutual information (CMI) adds the selection of redundant variables on the basis of variable correlation. On the premise of $S$, the importance of $x$ is determined by interdependency between $x$ and $y$. In other words, a higher value of CMI shows more new information $x$ can provide conditioned on $S$.

The CMI between $x$ and $y$ conditioned on $S$ is defined by the following equation:

$$
I(x, y \mid S)=\iint_{S} \int_{y} p(x, y, S) \log \frac{p(x, y \mid S)}{p(x \mid S) p(y \mid S)} d x d y d S
$$

If $x, y$ and $S$ are random variables, $p(x, y, S)$ is probability density function, $p(x, y \mid S), p(x \mid S), p(y \mid S)$ are conditional probability distribution function.

\section{B. Selection of Variables for SCR Denitrification System}

This paper took the SCR de-NOx system of coal-fired units as research object. The SCR reaction mechanism of coal-fired units was complex, and the influential factors of denitrification efficiency could be quite a lot. This paper selected genarator power $\left(x_{1}\right)$, total air volume $\left(x_{2}\right)$, total coal volume $\left(x_{3}\right)$, input NOx concentration $\left(x_{4}\right), \mathrm{NH}_{3}$ flow $\left(x_{5}\right), \mathrm{NH}_{3}$ valve position $\left(x_{6}\right)$, oxygen concentration in flue gas $\left(x_{7}\right)$ and flue gas temperature $\left(x_{8}\right)$ as original input variables, and output NOx concentration as the output variable $(y)$.

According to the process analysis of SCR de-NOx system, gas flow and chemical reaction could give rise to the time lag, in addition, the correlation and redundancy should be fully considered. Therefore, this paper proposed the following method:

\section{1) Selection of optimal time delay:}

a) Suppose 16 types of time delay for each of the 8 input variables (As a matter of experience, initial time delay is in the range of 0 to $150 \mathrm{~s}$. Sampling time is $10 \mathrm{~s}$, so $\tau \in[0,15])$.

b) Calculate the MI between $120(8 * 15)$ types of input sequences and the output variable, as is shown in (3). When every input variable and the output variable have the maximum MI, the delay is the optimal delay of corresponding input variable.

2) Selection of input variables:

a) Set threshold value $\delta=0.15(\delta I(y, y)=0.7)$, and use the following equation

$$
I\left(x_{i}(t-\tau), y\right)>\delta I(y, y)
$$

to eliminate weakly correlated variables: oxygen concentration in flue gas. The result is as shown in the Figure 2.

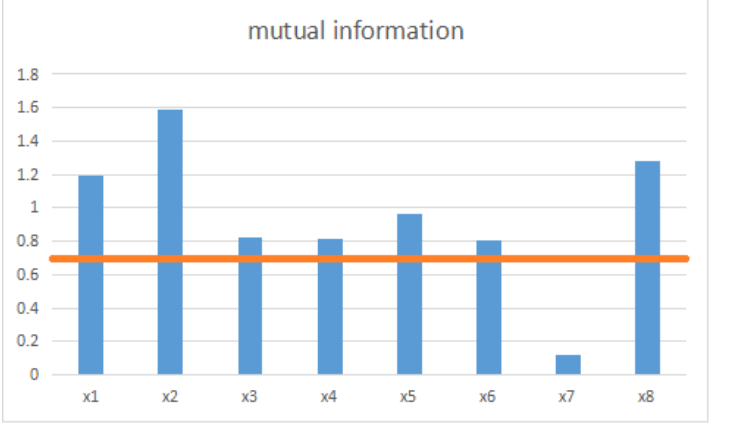

FIGURE II. $\quad$ PRELIMINARY SELECTION BASED ON MI

b) Initialize $S$ as a null set. Select the input sequence of the maximum $M I$ and put it in $S$.

c) On the premise of $S$, calculate the CMI (shown in (4)) between every input variable and the output variable, and the input variable with the largest CMI is selected and put into $S$.

d) Repeat the step c) until the observed value is zero, or the number of input variables meets the qualification. And the final selected input variables are the optimal ones. The experimental conditions and their results were summarized in Table 1.

TABLE I. THE OPTIMAL INPUT VARIABLES

\begin{tabular}{|l|l|l|}
\hline \multirow{2}{*}{ No } & \multicolumn{2}{|c|}{ Selected input variables } \\
\cline { 2 - 3 } & \multicolumn{1}{|c|}{ Name } & \multicolumn{1}{c|}{ Time delay (10s) } \\
\hline 1 & total air volume & -9 \\
\hline 2 & input NOx concentration & -2 \\
\hline 3 & NH3 flow & -8 \\
\hline 4 & flue gas temperature & -7 \\
\hline
\end{tabular}

The MI between the optimal input variables and the output in different delay time are shown in Figure 3. 


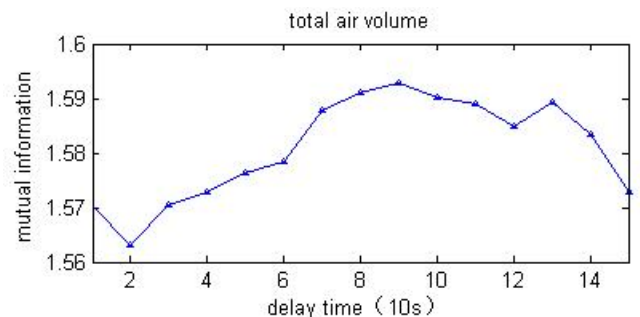

(a)

input NOx concentration

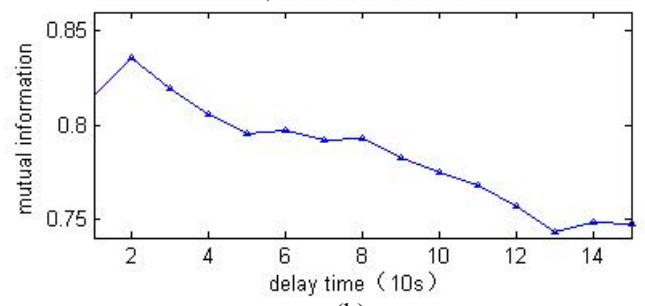

(b)

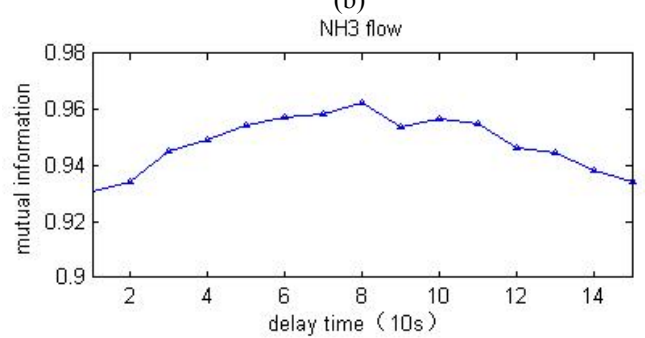

(c)

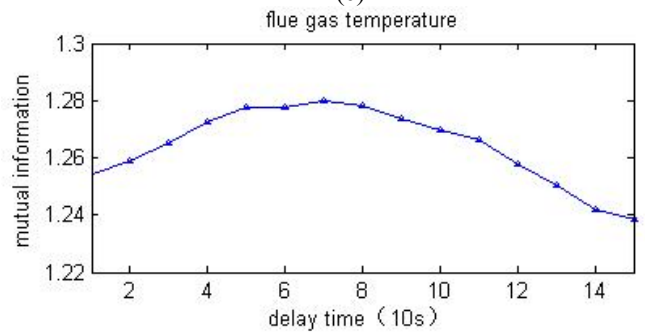

(d)

FIGURE III.

TIME DELAY ESTIMATION BASED ON MI

The total air volume, input $\mathrm{NOx}$ concentration and $\mathrm{NH}_{3}$ flow are selected, and they directly participate in chemical reactions, Temperature determines the speed of chemical reactions, therefore the results fit chemical principle well. Genarator power and total coal volume are eliminated because of the small value of the CMI, and they are highly redundant with total air volume in reality. Likewise, $\mathrm{NH}_{3}$ valve position is eliminated, as it is used to control $\mathrm{NH}_{3}$ flow. The method screens out variables of low interdependency and high redundancy well, and simplify subsequent modeling.

\section{DYNAMIC DATA MODELING}

\section{A. Least Squares Support Vector Machine}

As an intelligent soft computing method, LSSVM develops a comprehensive model with minimum structural risk to predict targets by using nonlinear kernel regression, and the regression function of LSSVM can be defined as follows:

$$
\left\{\begin{array}{l}
\min \frac{1}{2} \omega^{T} \omega+\frac{1}{2} \gamma \sum_{i=1}^{n}\left(\xi^{2}\right), \\
\text { s.t. } y=\omega^{T} \varphi\left(x_{i}\right)+b+\xi^{2}, i=1,2, \cdots, n
\end{array}\right.
$$

where $\omega$ is weight vector, $\varphi()$ is map function, $\gamma$ is penalty function, $\xi$ is slack variable.

The model of LSSVM is obtained by lagrange multiplier and KKT condition, and defined as follows :

$$
y(x)=\sum_{i=1}^{n} \alpha_{i} K\left(x, x_{i}\right)+b,
$$

where $\alpha_{i}$ is the lagrange multiplier, $K\left(x, x_{i}\right)$ is kernel function. This paper uses Radial basis Function (RBF) as kernel function:

$$
K\left(x, x_{i}\right)=\exp \left(-\left\|x-x_{i}\right\|^{2} / \sigma^{2}\right)
$$

\section{B. Prediction of Output NOx Concentration}

Based on the result of MI and LSSVM modeling, this paper selected total air volume, input NOx concentration, NH3 flow, flue gas temperature and the first order feedback from output NOx concentration as the input variables to predict. The structure of the model is shown in Figure 4.

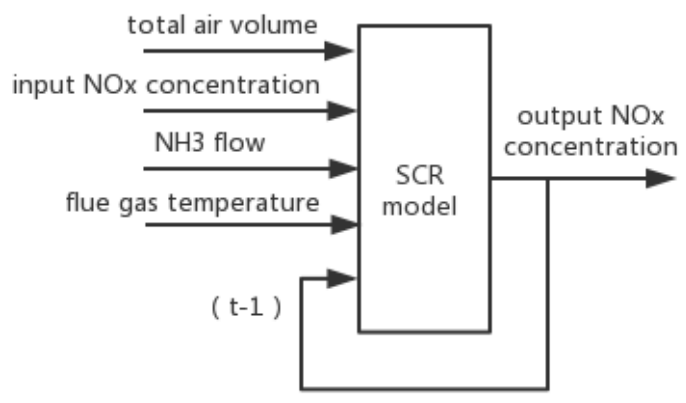

FIGURE IV.

THE STRUCTURE OF THE MODEL

This paper selected 8000 groups of the historical data in dynamic as samples, among which 6000 groups were used as the training samples while the rest were test ones. As for parameter optimization, particle swarm optimization (PSO) were used to search optimal parameters $\gamma$ and $\sigma^{2}$ for LSSVM. RMSE and MAE were used to evaluate the accuracy of the model. The formulas are as follows:

$$
R M S E=\sqrt{\sum_{i=1}^{n}\left(y_{i}-\hat{y}_{i}\right) / n},
$$




$$
M A E=\frac{1}{n} \sum_{i=1}^{n}\left|y_{i}-\hat{y}_{i}\right|
$$

Training, test curves and their errors are shown in Figure 5. As for RMSE and MAE, the training set: $\mathrm{RMSE}=0.3685$, $\mathrm{MAE}=0.1758$; test set: $\mathrm{RMSE}=1.0472, \mathrm{MAE}=0.7908$. These outcomes suggest that the model can be compatibly used for the modeling of SCR de-NOx system, which exists complex relationships between variables.

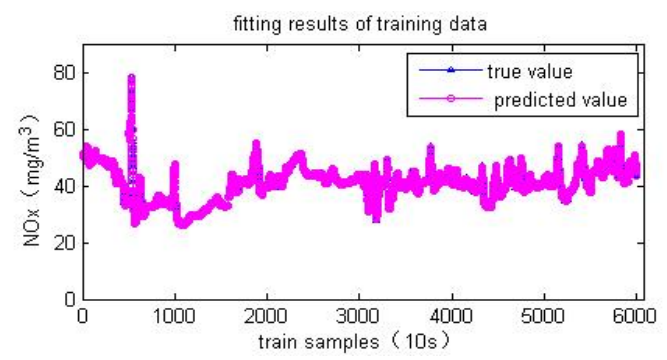

(a)

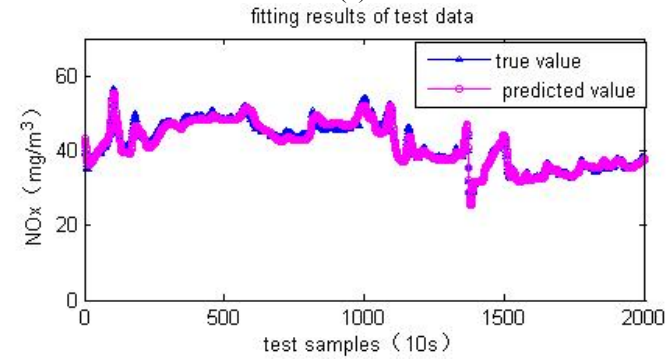

(b)

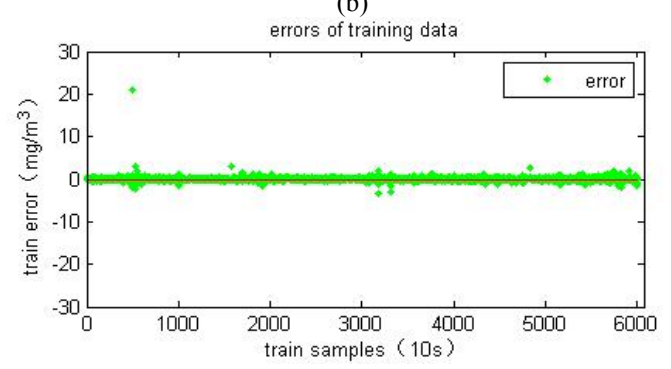

(a)

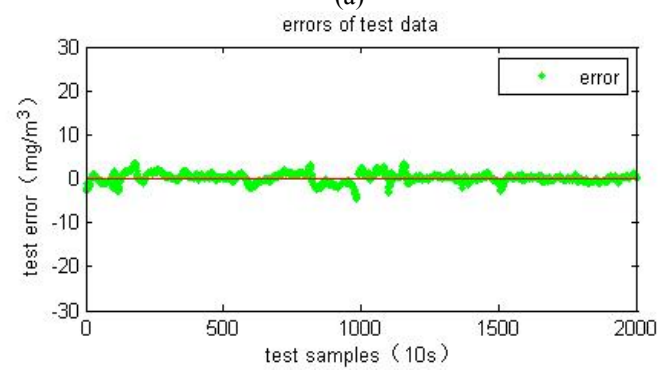

(b)

FIGURE V.

FITTED CURVE AND ERRORS

\section{CONCLUSION}

In view of the complex mechanism of SCR de-NOx system and the strong coupling between variables, this paper presents a dynamic data modeling method based on least squares support vector machine (LSSVM) and mutual information (MI) based on the SCR de-NOx data of a power plant. This method evaluated the importance of every input variable and chose the ones of high correlation and low redundancy as the inputs of LSSVM to calculate output NOx concentration. The results revealed that combination of LSSVM and MI can be successfully used in estimating the dynamic characteristis of output NOx concentration.

\section{ACKNOWLEDGMENT}

This project was funded by the National Key R\&D of China (Grant No: 2016YFB0600701) and the Hebei Province Natural Science Foundation of China (No. F2014502059). The authors would like to thank Engineer Geng B. of JingLong power plant for his assistance to collect the operation data.

\section{REFERENCES}

[1] N. Y. Topsøe, "Mechanism of the Selective Catalytic Reduction of Nitric Oxide by Ammonia Elucidated by in Situ On-Line Fourier Transform Infrared Spectroscopy," Science, vol. 265, pp. 1217-1219, August 1994.

[2] H. Zhou, K. Cen, and J. Fan, "Modeling and optimization of the NOx emission characteristics of a tangentially fired boiler with artificial neural networks," Energy, vol. 29, pp. 167-183, January 2004.

[3] F. Si, et al. "Optimization of coal-fired boiler SCRs based on modified support vector machine models and genetic algorithms." Fuel, vol. 88, pp. 806-816, May 2009.

[4] T. Yang, C. Cui, Y. Lv, and J. Li, "Modeling on SCR process of a coalfired boiler using LSSVM method," IEEE Control and Decision Conference, 2015, pp. 4025-4028.

[5] S. Chehreh Chelgan, B. Shahbazi, and E. Hadavandi, "Support vector regression modeling of coal flotation based on variable importance measurements by mutual information method," Measurement, vol. 114, pp. 102-108, January 2018.

[6] M. Han, W. Ren, and X. Liu, "Joint mutual information-based input variable selection for multivariate time series modeling," Engineering Applications of Artificial Intelligence, vol. 37, pp. 250-257, January 2015.

[7] F. Kaytez, M. C. Taplamacioglu, E. Cam, and F. Hardalac, "Forecasting electricity consumption: A comparison of regression analysis, neural networks and least squares support vector machines," International Journal of Electrical Power \& Energy Systems, vol. 67, pp. 431-438, May 2015.

[8] G. Brown, A. Pocock, M. Zhao, and M. Luján, "Conditional Likelihood Maximisation: A Unifying Framework for Information Theoretic Feature Selection," Journal of Machine Learning Research, vol. 13, pp. 27-66, January 2012. 